COPYRIGHT @ 2019 INTERNATIONAL JOURNAL OF SCIENCE DENTISTRY | AVAILABLE ONLINE

http://www.periodicos.uff.br/index

\title{
TRATAMENTO DE LUXAÇÃO MANDIBULAR CRÔNICA COM EMINECTOMIA: RELATO DE CASO
}

\section{TREATMENT OF CHRONIC MANDIBULAR DISLOCATION WITH \\ EMINECTOMY: CASE REPORT}

Eugênio Rodrigues Arantes

Residente de Cirurgia Oral e Maxilofacial do Hospital Federal dos Servidores do Estado do Rio de Janeiro - HFSE e da Universidade Federal Fluminense - UFF.

Suelen Cristina Sartoretto

Professora das disciplinas de Cirurgia Bucal e Anatomia da Faculdade de Odontologia da Universidade Iguaçu - UNIG.

Fernanda Britto de Melo Silva

Residente de Cirurgia Oral e Maxilofacial do Hospital Federal dos Servidores do Estado do Rio de Janeiro - HFSE e da Universidade Federal Fluminense - UFF.

Marcelo José Uzeda

Professor da Disciplina de Cirurgia Bucal da Universidade Federal Fluminense - UFF e Professor das disciplinas de Cirurgia Bucal e Anatomia da Faculdade de Odontologia da Universidade Iguaçu - UNIG.

Instituição na qual o trabalho foi realizado: Hospital Federal dos Servidores do Estado do Rio de Janeiro/RJ

Categoria: Relato de caso

\section{Informações do autor principal:}

Eugênio Braz Rodrigues Arantes

Rua Mário Santos Braga, 28

Centro - Niterói - RJ, Brasil.

CEP: 24020-140

E-mail: ebrarantes@gmail.com

Telefone: +552126299920 
COPYRIGHT @ 2019 INTERNATIONAL JOURNAL OF SCIENCE DENTISTRY | AVAILABLE ONLINE

http://www.periodicos.uff.br/index

\title{
Resumo
}

A luxação da articulação temporomandibular é caracterizada quando o côndilo ultrapassa os movimentos limítrofes da sua excursão normal e se desloca para fora da cavidade glenoide, à frente da eminência articular, permanecendo temporariamente retido. A recorrência dessa patologia caracteriza uma condição recidivante ou crônica. As formas de tratamento para essa desordem estão de acordo com a periodicidade e o tempo de evolução e podem variar desde tratamentos conservadores locais até procedimentos cirúrgicos invasivos. O objetivo do presente trabalho é apresentar o relato de um caso clínico de uma paciente com luxação recidivante da ATM tratada através da técnica de remoção da eminência articular, também chamada de eminectomia. O tratamento deste caso através de eminectomia mostrou-se eficiente e adequado em relação à abertura máxima, recidiva e função articular pós-operatória.

Palavras-chave: Articulação temporomandibular, transtornos da articulação temporomandibular, luxações articulares, artroplastia.

\begin{abstract}
Temporomandibular joint dislocation occurs when the condyle exceed the borderline movements of its normal excursion and moves out of the glenoid cavity, remaining temporarily retained in front of the articular eminence. The recurrence of this pathology characterizes a relapsing or chronic condition. The forms of treatment for this disorder are according to the periodicity and time of evolution and may range from local conservative treatments to invasive surgical procedures. The aim of the present paper is to present a case report of a patient with recurrent TMJ dislocation treated by the joint eminence removal technique, also called eminectomy. The proposed treatment in this case proved to be efficient and adequate in relation to the maximum mouth opening, recurrence and postoperative joint function.
\end{abstract}

Key-words: Temporomandibular joint, temporomandibular joint disorders, joint dislocations, arthroplasty. 


\section{Introdução}

A luxação da articulação temporomandibular é definida como o deslocamento do côndilo mandibular além da fossa glenoide sem a habilidade de redução espontânea. Esta patologia apresenta-se de forma aguda ou crônica e com deslocamento para anterior, posterior, superior, medial ou lateral, sendo o deslocamento anterior considerado o mais comum e os outros tipos estão normalmente associados à fraturas (PRECHEL U, OTTL P, ET AL. 2018).

Essa condição afeta normalmente adultos jovens, bilateralmente, incapacitando o movimento de fechar a boca, devido ao restrito movimento mandibular em ambos os lados da articulação temporomandibular (ATM). Além disso, são identificados espasmos musculares no grupamento de músculos da mastigação associados à dor na região pré-auricular bilateral (PRECHEL U, OTTL P, ET AL. 2018; MATEO MM, TORRES MP, ET AL. 2016). Os fatores etiológicos são múltiplos e o trauma ou a abertura excessiva da mandíbula são os principais fatores relacionados. As causas intra-articulares podem ser explicadas por um desenvolvimento ósseo deficiente da fossa articular, flacidez dos ligamentos da articulação temporomandibular ou atividade muscular excessiva (MATEO MM, TORRES MP, ET AL. 2016).

Em alguns casos, a redução espontânea geralmente é resolutiva, considerada subluxação, no entanto, há casos em que os episódios se tornam recorrentes e evoluem para uma patologia crônica. $\mathrm{O}$ tratamento varia de métodos conservadores à intervenções cirúrgicas (MATEO MM, TORRES MP, ET AL. 2016; PAPOUTSIS G, PAPOUTSI S, ET AL. 2018), objetivando limitar a excursão côndilo mandibular anteriormente a eminência. Abordagens como infiltração intracapsular de soluções esclerosantes, bloqueio aloplástico com implantes ou placas de titânio, redução da eminência articular, permitindo a livre movimentação do côndilo, são descritas na literatura com suas vantagens e desvantagens (PAPOUTSIS G, PAPOUTSI S, ET AL. 2018).

Dessa forma, o objetivo do presente estudo é apresentar o relato de um caso clínico de luxação crônica da ATM tratada através da remoção da eminência articular, também conhecida como eminectomia bilateral.

\section{Considerações Éticas}

REVISTA FLUMINENSE DE ODONTOLOGIA - ANO XV - № 51 - Janeiro / Junho 2019 
Os procedimentos empregados seguiram os padrões éticos da Declaração de Helsinque de 1975, revisada em 2000. A paciente foi submetida a tratamento cirúrgico no Hospital Federal dos Servidores do Estado do Rio de Janeiro conveniado com a Universidade Federal Fluminense através da Residência em Cirurgia Oral e Maxilofacial, após assinar o termo de consentimento livre e esclarecido para uso de imagens e dados para publicação científica.

O estudo foi conduzido de acordo com as Diretrizes do Relatório de Caso Clínico (CARE, www.care-statement.org).

\section{Relato de caso}

Paciente N.F.S, 44 anos de idade, sexo feminino, com o diagnóstico de luxação crônica da articulação temporomandibular, procurou o Serviço de Cirurgia Oral e Maxilofacial do Hospital Federal dos Servidores do Estado do Rio de Janeiro, queixando-se de dor e episódios recorrentes de luxação mandibular com tempo de evolução de cinco anos. A queixa principal era de "mandíbula caindo e não conseguir voltar ao normal".

Durante a anamnese a paciente relatou ter sido submetida a um procedimento cirúrgico prévio sem resultado satisfatório para instalação de fios de aço bilateralmente na região anterior à eminência articular com o objetivo de limitar o seu movimento condilar e, consequentemente, impedir os episódios repetidos da luxação mandibular.

Ao exame intra-bucal apresentava ausências dentárias e uso de próteses removíveis. A avaliação por imagem realizada através de radiografia panorâmica dos maxilares demonstrou eminência articular pronunciada e fossa articular profunda em ambos os lados além da presença de fios de aço bilateralmente confirmando o histórico cirúrgico para tentativa de tratamento inicial (Figura 1 A).

No planejamento cirúrgico optou-se pela remoção da eminência articular com a finalidade de permitir o livre movimento condilar e retirada dos materiais utilizados na primeira cirurgia. O procedimento cirúrgico foi realizado sob anestesia geral e intubação naso-traqueal. Foram feitas marcações das linhas de incisão e infiltração com lidocaína $2 \%$ e epinefrina 1:100.000. O acesso de Al Kayat foi realizado para melhor visualização da área correspondente à eminência articular se estendendo até a região temporal (Figuras 2 A e B). A broca cirúrgica 702 foi utilizada para marcação e realização da osteotomia da eminência articular. A clivagem foi finalizada com cinzel reto e a osteoplastia realizada com broca para 
desgaste. No trans-cirúrgico foi possível simular os movimentos de abertura e fechamento da boca para visualizar a excursão correta e sem interferências dos côndilos mandibulares de ambos os lados (Figuras 2 C e D).

Durante o acompanhamento pós-operatório observou-se o aumento da abertura bucal e ausência de episódios de luxação mandibular. Em um acompanhamento pós-operatório de 24 meses, não houve recidiva e a mesma segue sem alterações clínicas ou por imagem (Figura 1 B). A paciente apresenta um índice de abertura bucal máxima adequado de aproximadamente 37 mm (Figura 3).
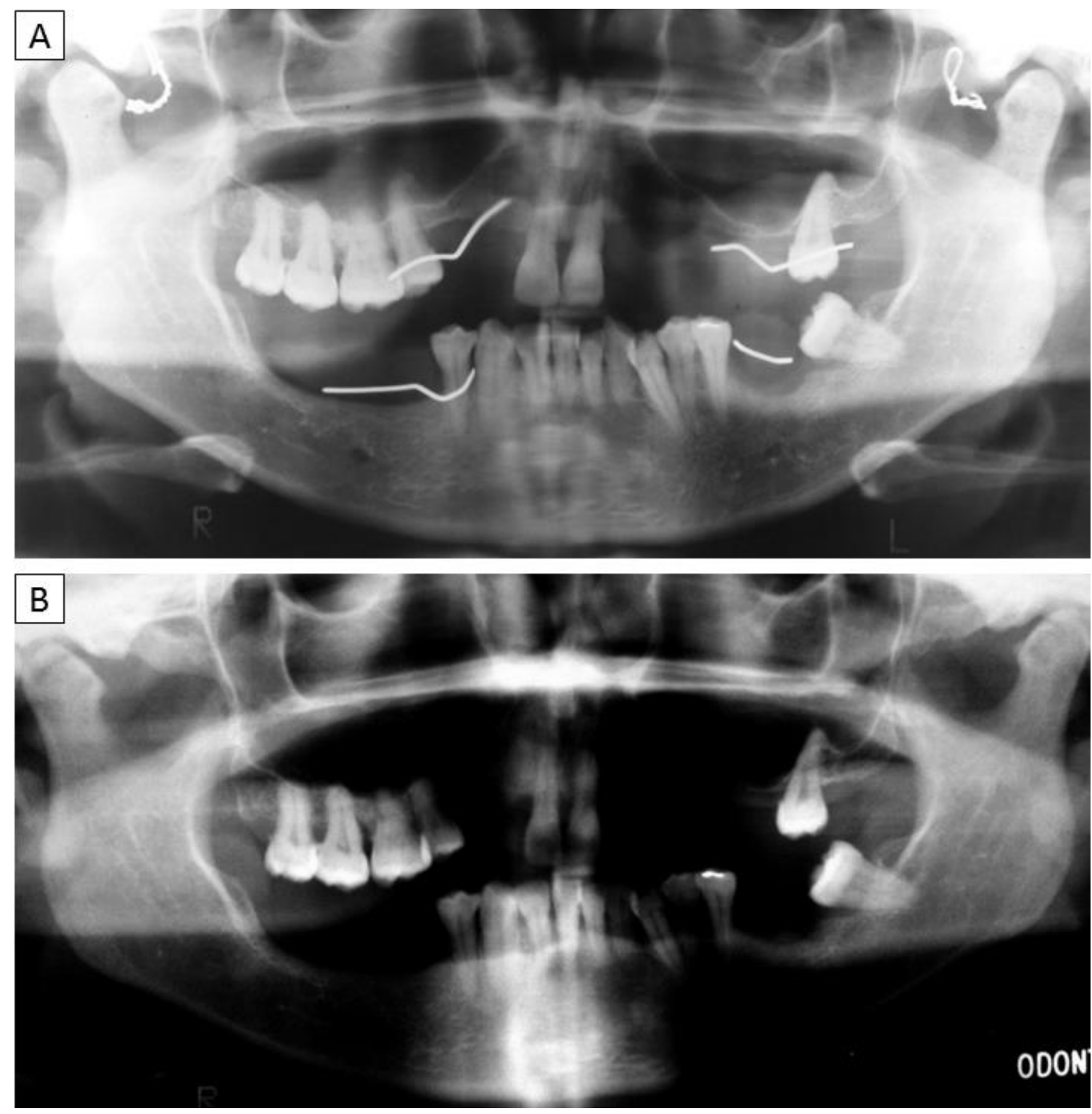
Figura 1. A) Radiografia panorâmica inicial evidenciando eminências articulares de ambos os lados após a primeira intervenção cirúrgica. B) Radiografia panorâmica final após procedimento cirúrgico definitivo com as eminências articulares aplainadas.
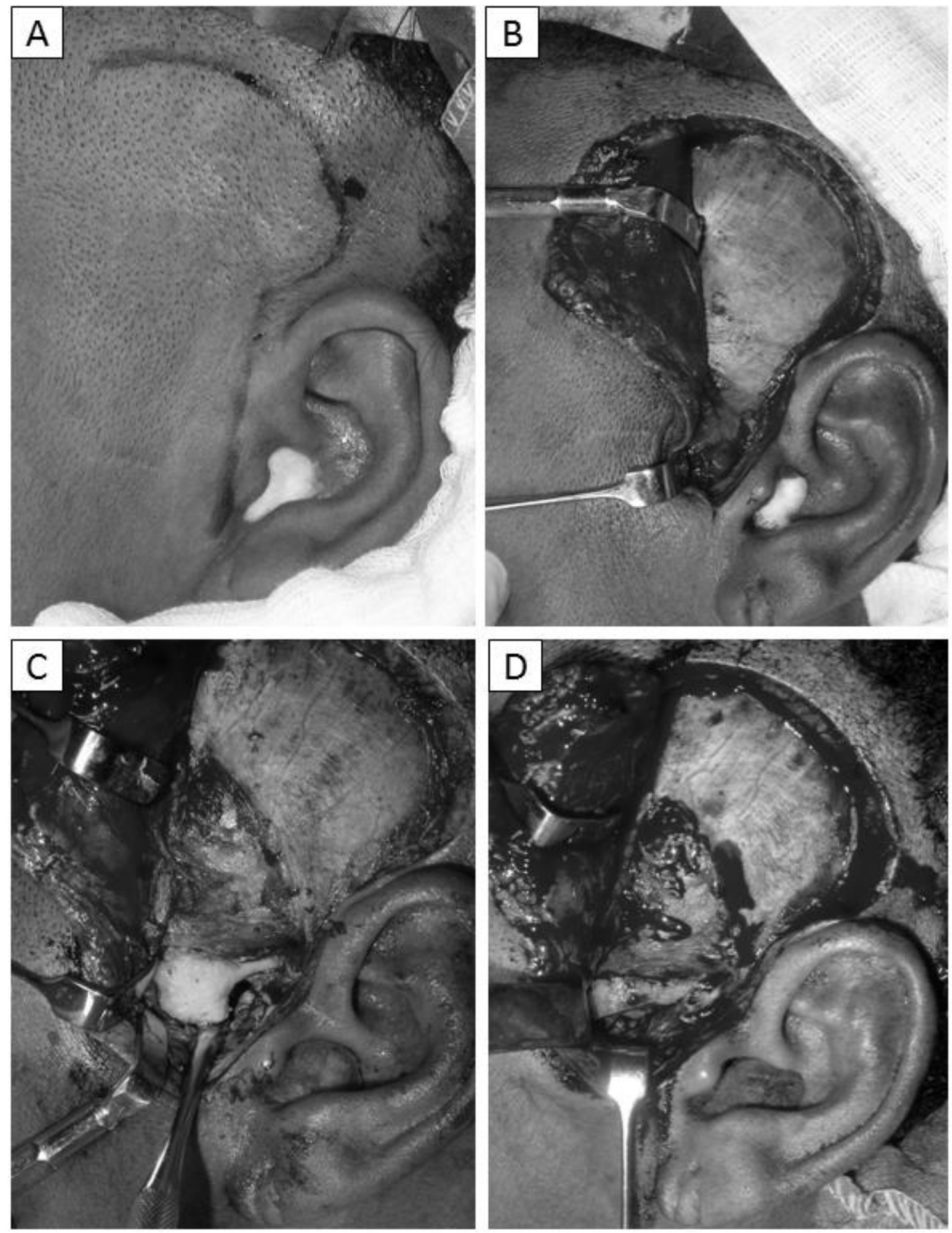

Figura 2: Procedimento cirúrgico. A) Marcação da área de incisão para acesso cirúrgico pré-auricular com extensão (Al Kayat). B) Acesso à região temporal e a REVISTA FLUMINENSE DE ODONTOLOGIA - ANO XV - № 51 - Janeiro / Junho 2019 
cápsula da articulação temporomandibular. C) Articulação temporomandibular com eminência articular proeminente. D) Aspecto final da eminência articular após osteotomia.
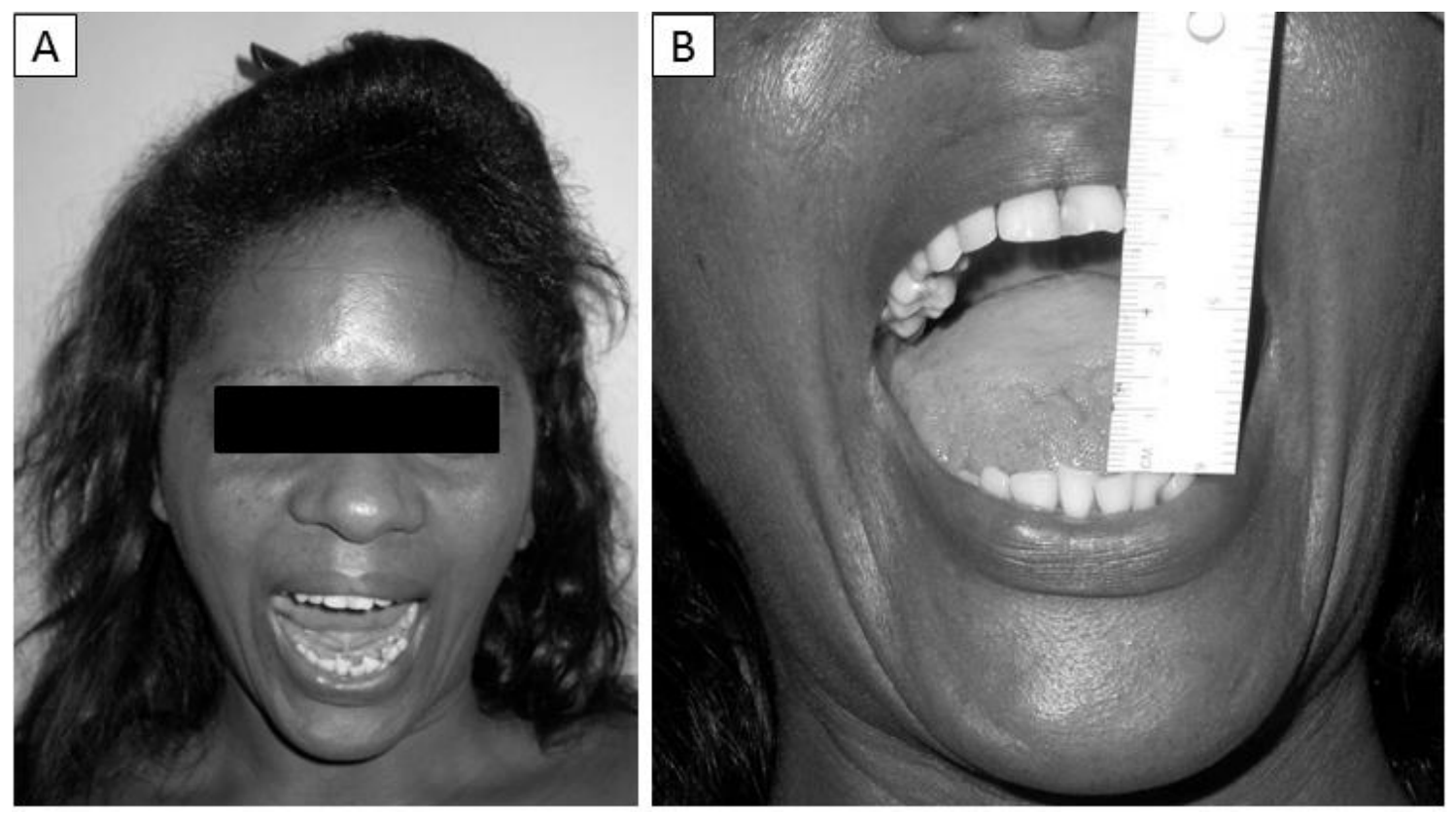

Figura 3. A) Fotografia final demonstrando boa movimentação durante abertura de boca. B) Índice de abertura de boca com boa amplitude (aproximadamente $37 \mathrm{~mm}$ ).

\section{Discussão}

Em 1993 duas modalidades cirúrgicas para a luxação recidivante da ATM foram apresentadas como forma de tratamento para esta desordem, em ambas, o objetivo principal era diminuir os episódios recorrentes do deslocamento mandibular (PUELACHER WC, WALDHART E. 1993). O primeiro método terapêutico objetivava restringir a abertura bucal, através de um aumento da eminência articular com o auxílio de materiais de síntese óssea (fios de aço ou placas, parafusos e implantes) gerando um obstáculo mecânico no trajeto condilar. O segundo método permitia movimentos mandibulares livres através da remoção da eminência articular, procedimento esse conhecido nos dias atuais por eminectomia (MATEO MM, TORRES MP, ET AL. 2016; PUELACHER WC, WALDHART E. 1993).

A instalação de miniplacas ou fios de aço em um segmento específico da eminência articular visa prevenir a hiperexcursão do côndilo, impedindo o seu deslocamento (MARTINS WD, RIBAS MO, ET AL. 2014; AKINBAMI BO. 2011). Trata-se de uma técnica considerada menos invasiva, reversível e que promove resultados imediatos uma vez que o movimento 
mandibular é logo limitado pelas restrições mecânicas proporcionadas pelo material instalado (BUCKLEY MJ, MERRILL RG, ET AL. 1993). No caso descrito, a paciente foi inicialmente tratada através de uma abordagem cirúrgica para instalação de fios de aço na eminência articular bilateralmente, porém, após deformação do fio, a mesma retornou com os episódios de luxação, sendo necessária a retirada do material e novo planejamento cirúrgico. Na literatura, essa falha já havia sido relatada, uma vez que fraturas de placa ou deformação dos fios de aço podem acontecer durante os movimentos de excursão mandibular, exigindo novas intervenções cirúrgicas. Além disso, esse método diminui o grau de amplitude bucal, fato considerado uma grande desvantagem da técnica (VASCONCELOS BCE, PORTO GG. 2009).

O procedimento cirúrgico de remoção da eminência articular promove a manutenção dos movimentos articulares normais, não paralisados, simulando a função fisiológica. Ele consiste na remoção da acentuada inclinação da eminência articular, que previamente impedia o retorno do côndilo mandibular para a fossa glenoide, permitindo os movimentos mandibulares livres e sem o risco de luxação (PRECHEL U, OTTL P, ET AL. 2018; VASCONCELOS BCE, PORTO GG. 2009). As principais vantagens descritas na literatura para esta técnica são impedir os episódios de recorrência das luxações e promover a função articular normal logo no pós-operatório imediato (AKINBAMI BO. 2011). Alguns sinais e sintomas podem ser relatados pelos pacientes no pós-operatório imediato do procedimento da eminectomia, como discreta diminuição na abertura máxima da boca, crepitações e estalidos e dor (PRECHEL U, OTTL P, ET AL. 2018; MATEO MM, TORRES MP, ET AL. 2016).

A frouxidão nas estruturas articulares internas causa perda de coordenação no complexo articular ocasionando um desarranjo interno com consequente estiramento dos ligamentos articulares, fato esse que gera um desequilíbrio nos movimentos funcionais da mandíbula e, por este motivo, a eminectomia, é normalmente mais eficaz para o tratamento definitivo (VASCONCELOS BCE, PORTO GG. 2009; MARTINS WD, RIBAS MO, ET AL. 2014). No caso clínico descrito, a eminectomia pôde propiciar a livre movimentação do côndilo mandibular, eliminou a dor na articulação e melhorou o funcionamento da ATM. No entanto, a remoção da eminência pode levar à hipermobilidade e abertura excessiva da boca, o que pode desenvolver em longo prazo, degeneração da articulação (VASCONCELOS BCE, PORTO GG. 2009).

Outros métodos de tratamento mais conservadores visam restringir o movimento do côndilo com a injeção de substâncias esclerosantes levando a indução de fibrose nos tecidos REVISTA FLUMINENSE DE ODONTOLOGIA - ANO XV - No 51 - Janeiro / Junho 2019 
adjacentes à articulação (PUELACHER WC, WALDHART E. 1993). A injeção de sangue do próprio paciente também pode levar a formação de um coágulo organizado e consequente tecido fibroso, que pode manter os movimentos articulares limitados. O uso destas substâncias, como a toxina botulínica, como tratamento para a luxação da ATM, ainda apresentam eficácia controversa. De certa forma, os métodos conservadores induzem um alívio temporário dos sinais e sintomas, podendo levar novos episódios de recorrência (MARTINS WD, RIBAS MO, ET AL. 2014; AKINBAMI BO. 2011).

\section{Conclusão}

A técnica de eminectomia apresenta bons resultados ao ser indicada de maneira correta para os casos de luxação temporomandibular devido a sua fácil execução e pós-operatório imediato consistente. A manutenção dos movimentos funcionais normais da mandíbula e a ausência de recidiva fazem desta técnica cirúrgica a primeira opção de escolha.

\section{Referências}

1. Prechel U, Ottl P, et al. The treatment of temporomandibular joint dislocation. Dtsch Arztebl Int. 2018;115(5):59-64.

2. Mateo MM, Torres MP, et al. Temporomandibular chronic dislocation: the longstanding condition. Med Oral Patol Oral Cir Bucal. 2016;21:776-783.

3. Papoutsis G, Papoutsi S, et al. Temporomandibular joint dislocation: a retrospective study from a Swiss urban emergency department. Open Access Emerg Med. 2018;10:171-176.

4. Puelacher WC, Waldhart E. Miniplate eminoplasty: a new surgical treatment for TMJdislocation. J Craniomaxillofac Surg. 1993;21:176-8.

5. Martins WD, Ribas MO, et al. Recurrent dislocation of the temporomandibular joint: A literature review and two case reports treated with eminectomy, CRANIO. 2014; 32:2, $110-117$.

6. Akinbami BO. Evaluation of the mechanism and principles of management of temporomandibular joint dislocation: Systematic review of literature and a proposed new classification of temporomandibular joint dislocation. Head Face Med. 2011;7:10.

7. Buckley MJ, Merrill RG, et al. Surgical management of internal derangement of the temporomandibular joint. J Oral Maxillofac Surg. 1993; 51 (1 Supl): 20S a 27S. 
8. Vasconcelos BCE, Porto GG. Treatment of chronic mandibular dislocations: A comparison between eminectomy and miniplates. J Oral Maxillofac Surg. 2009; 67:2599-2604. 